\title{
Absence of First-Order Phase Transition in SU(5) Lattice Gauge Theory with Renormalization-Group-Improved Action
}

\author{
S. Itoh, Y. Iwasaki, and T. Yoshié \\ Institute of Physics, University of Tsukuba, Ibaraki 305, Japan \\ (Received 22 May 1985)
}

\begin{abstract}
Using Monte Carlo methods, we conclude that there are no first-order phase transitions in SU(5) lattice gauge theory with a renormalization-group-improved action, the form of which has been proposed previously by a block-spin renormalization-group analysis. This is quite different from the situation with the standard one-plaquette action.
\end{abstract}

PACS numbers: $11.15 . \mathrm{Ha}, 05.50 .+\mathrm{q}$

A few years ago a first-order phase transition was observed by Monte Carlo (MC) simulations for SU(5) lattice gauge theory in four dimensions with the standard one-plaquette action, ${ }^{1,2}$ as well as for $\mathrm{SU}(4)^{3,4}$ and SU(6) ${ }^{5}$ lattice gauge theories. It is believed to be an artifact of the one-plaquette form of the action. ${ }^{6}$ If one takes a lattice action which is close to the renormalized trajectory (RT) of a renormalization group (RG), there will be no first-order phase transitions, because the actions on a RT describe the essentially same system only with different scales. ${ }^{7,8}$ (The discussion on the arbitrariness of choice of $R G$ will be given later.) We have proposed such a lattice action, which will be referred to as RG-improved action, from a perturbative block-spin $R G$ analysis ${ }^{9}$ and from a study of the stability of instantons on the lattice. ${ }^{10}$ In this Letter we will report that there are indeed no first-order phase transitions for SU(5) lattice gauge theory with this RG-improved action. This result verifies the conjecture that the observed phase transition is an artifact of the standard one-plaquette action, and it supports also the strategy described in Ref. 9 to obtain a $\mathrm{RG}$-improved action.

We take a four-dimensional hypercubic lattice with periodic boundary conditions. The lattice size is $4^{4}$. We associate with the link a unitary unimodular $5 \times 5$ matrix. The action is

$$
\begin{array}{r}
S=g^{-2}\left[c_{0} \sum \operatorname{Tr}(\text { simple plaquette loop })\right. \\
\left.+c_{1} \sum \operatorname{Tr}(\text { rectangular loop })\right],
\end{array}
$$

with

$$
c_{1}=-0.331, \quad c_{0}=1-8 c_{1} .
$$

In a sum over loops, each oriented loop appears once. We use the algorithm by Cabibbo and Marinari ${ }^{11}$ to generate gauge-field configurations. In each step of the iteration the new link variable $U_{n, \mu}^{\prime}$ is obtained by multiplying the previous value $U_{n, \mu}$ by ten matrices as follows:

$$
U_{n, \mu}^{\prime}=\prod_{i>j} a_{i j} U_{n, \mu} \quad(1 \leqslant i, j \leqslant 5),
$$

where $a_{i j}$ is an element of the SU(2) subgroup such that the $i$ th and the $j$ th rows and columns of the $5 \times 5$ matrix compose an SU(2) matrix. One iteration by this method will correspond to at least several iterations by the Metropolis method such as employed in Refs. 1 and 2, if we guess from the known result for SU(3) gauge theory concerning the difference between Metropolis method and Cabbibo-Marinari method. This conjecture will be verified later.

We begin with a rapid thermal cycle with a given action, measuring the value of the one-plaquette Wilson loop,

$$
W(1 \times 1)=\frac{1}{5}\left\langle\operatorname{Tr} U_{\square}\right\rangle,
$$

in order to guess a possible position where a first-order phase transition occurs, if it exists at all. Here $U_{\square}$ is an ordered product of the link variable around a plaquette. Then we perform 100 iterations, starting both from a completely ordered (cold) state and from a completely disordered (hot) state at several fixed $\beta$ 's $\left(\beta=10 / g^{2}\right)$, with steps $\Delta \beta=0.1$ around the possible phase-transition point.

We first make the process described above for the standard one-plaquette action, because our updating procedure is different from that in Refs. 1 and 2, in order to check whether our procedure works well for probing the first-order phase transition. We perform 100 iterations described above for $16.1 \leqslant \beta \leqslant 16.8$ with steps $\Delta \beta=0.1$. We clearly see the existence of two states for $16.4 \leqslant \beta \leqslant 16.7$. The mean values of the one-plaquette Wilson loop for the last fifty iterations are shown in Fig. 1. We see a clear signal for the first-order phase transition which was observed in Refs. 1 and 2 . We make a further 300 iterations in total at $\beta=16.6$; the values of the one-plaquette Wilson loop versus the number of iterations are shown in Fig. 2. From Figs. 1 and 2 we conclude that $\beta_{c}=16.55$ \pm 0.15 , which is consistent with the value quoted in Ref. 1 .

If we compare our Fig. 2 with Fig. 1 of Ref. 1, we see the difference for the rate of convergence to equilibrium. This verifies the conjecture that one iteration by Cabbibo-Marinari method corresponds to at least several iterations by the Metropolis method employed in Ref. 1 . 


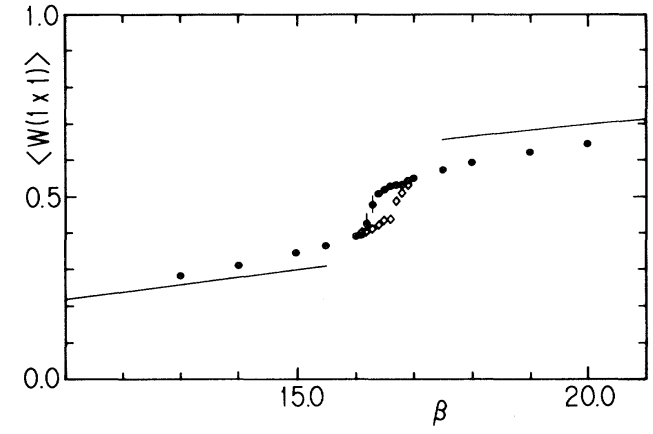

FIG. 1. The average value of the one-plaquette Wilson loop for the standard action as a function of $\beta$. The filled circles and open diamonds represent the average over the last 50 of 100 iterations through the lattice with cold and hot starts, respectively. The curves represent the leading-order strong- and weak-coupling expansions.

Next we do the same thing as above for the RG improved action in the range $5.5 \leqslant \beta \leqslant 6.4$, with steps $\Delta \beta=0.1$. After thirty iterations there are no noticeable differences between the values of the oneplaquette Wilson loop from a hot start and from a cold start, for all $\beta$ 's investigated. We represent the average of the one-plaquette Wilson loop for the last fifty iterations in Fig. 3. Although we plot values for both hot start and cold start, we are unable to see a difference between them with a precision of the size of the marks except for $\beta=6.0$. In the figure we also plot the averages of the one-plaquette Wilson loops at some values of $\beta$ for $\beta>6.5$ and $\beta<5.5$, together with the leading-order strong-coupling expansion and the leading-order weak-coupling expansion. The data for $\beta>6.5(\beta<5.5)$ are in good agreement with the weak- (strong-) coupling expansion:

$$
\begin{aligned}
& W(1 \times 1)=1-2.523 / \beta, \\
& W(1 \times 1)=0.0730 \beta .
\end{aligned}
$$

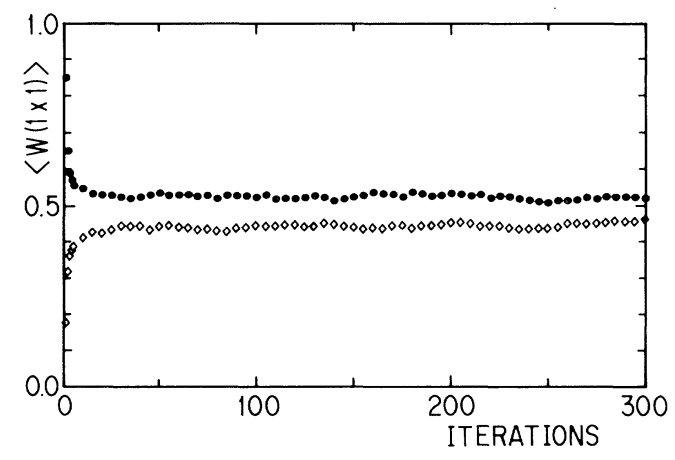

FIG. 2. The evolution of the one-plaquette Wilson loop of the ordered (circles) and the disordered (diamonds) states for the standard action at $\beta=16.6$.

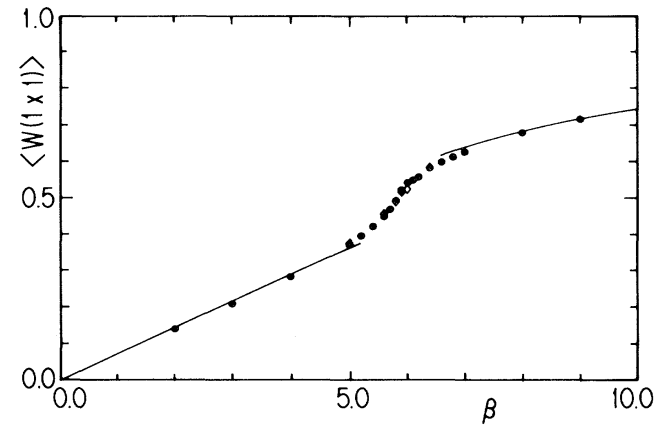

FIG. 3. The same as Fig. 1, except for the RG-improved action.

The rate of convergence from hot start and cold start to the common value seems to be slowest around $\beta=6.0$. Therefore we make in total 300 iterations at $\beta=6.0$. The results are shown in Fig. 4. As seen from the figure, we have no sign of a first-order phase transition. Even if we use the value of the action instead of the value of the one-plaquette Wilson loop as a probe, the results are essentially the same as previous ones. Thus we conclude that there are no first-order phase transitions for the RG-improved action, as conjectured already in Ref. 9.

Strictly speaking, we cannot draw in general a definite conclusion on the phase transition of an infinite system from a numerical study on a finite lattice. Probably, we have to state moderately that even if a first-order phase transition exists for the RG-improved action, the gap of the value of the one-plaquette Wilson loop is very small and is less than 0.01. Anyway, calculations of physical quantities by MC simulations are performed on a finite lattice, and therefore we conclude that for MC calculations on a finite lattice we may assume the nonexistence of first-order phase transitions for SU(5) lattice gauge theory, if we use the RG-improved action.

We also make a similar investigation for the action with $c_{1}=-\frac{1}{12}$ and $c_{0}=1-8 c_{1}$, which was proposed as

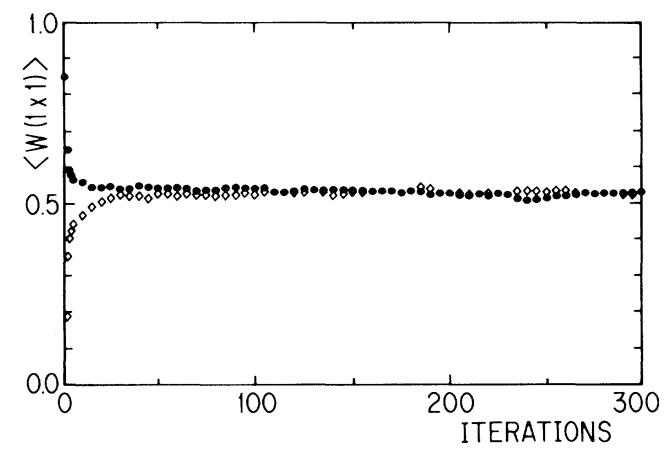

FIG. 4. The same as Fig. 2, except for the RG-improved action at $\beta=6.0$. 
TABLE I. The averages of the one-plaquette Wilson loop with the statistical errors for the last 220 of 300 iterations from both cold and hot initial states in the case of the three actions at some fixed $\beta$ 's. See text for more details.

\begin{tabular}{lcccc}
\hline \hline Action & Beta & Initialization & $\langle W(1 \times 1)\rangle$ & Error \\
\hline Standard & \multirow{2}{*}{16.6} & Cold & 0.5275 & 0.0012 \\
& & Hot & 0.4479 & 0.0015 \\
Improved & \multirow{2}{*}{6.0} & Cold & 0.5333 & 0.0017 \\
& & Hot & 0.5340 & 0.0009 \\
Symanzik & \multirow{2}{*}{11.3} & Cold & 0.4918 & 0.0018 \\
& & Hot & 0.4868 & 0.0012 \\
\hline \hline
\end{tabular}

an improved action ${ }^{12}$ in Symanzik's scheme. ${ }^{13}$ Because the numerical value $c_{1}=-\frac{1}{12}$ is between $c_{1}=0$ and $c_{1}=-0.331$, we may expect that the situation will also be between those observed for the standard action and for the RG-improved action. In Table I we list the averages of the one-plaquette Wilson loop with the statistical errors at $\beta=11.3$ for the last 220 iterations, disregarding the first eighty iterations starting both from a hot state and from a cold state. Presented there are also those for the other two actions. The errors are estimated by dividing the whole iterations into bunches of ten iterations, measuring the standard deviation of the bunch averages from the total average, and dividing it by the square root of the number of bunches. From Table I we see that the difference between the one-plaquette averages from hot start and from cold start is much larger than the statistical errors for the standard action at $\beta=16.6$, and the difference is less than the errors for the RG-improved action at $\beta=6.0$. On the other hand, for Symanzik's improved action the difference is slightly larger than the statistical errors at $\beta=11.3$. Thus, although we cannot say that there is a first-order phase transition, neither do we say definitely that there are no phase transitions.

Let us finally make a few comments:

(i) The definition of the RG is not unique, of course, and therefore the RT is not unique. There are a variety of transformations which can be adopted as a RG. To find $\Delta \beta$ for a scale change, many of them will work well as described by Bowler et al. ${ }^{14}$ However, if one wants to decrease lattice artifacts at small distances for the actions on the RT as small as possible, there will be certain criteria to choose the RG, although such criteria are not known yet. At least the actions on the RT should be effective actions for long-distance behavior of any lattice action such as the standard one-plaquette action. In Ref. 9, we have made an effort to find such a lattice action and have chosen the action represented by Eq. (1). Although the absence of first-order phase transitions only weakly supports the validity of our strategy, we have also found that the lattice spacing determined from the string tension is in remarkable agreement with that determined from meson spectrum ${ }^{15}$ with a RG-improved $\mathrm{SU}(3)$ action. This action is the same as the action (1), only with the modification of the gauge group from SU(5) to SU(3). (See Ref. 15 for more details.) We believe that these two facts together support rather strongly the validity of our strategy.

(ii) The RG-improved action is obtained by a perturbative block-spin RG analysis. Therefore it is guaranteed that the action is close to the RT only near the critical point. However, a possible point for a first-order phase transition is the crossover region from strong-coupling region to weak-coupling region and around the point where the mean value of the one-plaquette Wilson loop is 0.5 . For the RGimproved action this point is around $\beta=6.0$. Because the numerical value $\beta=6.0$ is rather large, we may expect that the action at $\beta=6.0$ is close to the RT. Our numerical results are consistent with this conjecture.

The numerical calculations have been performed with HITAC M280H at the National Laboratory for High Energy Physics (KEK). We would like to thank Hirotaka Sugawara and other members of KEK for their kind hospitality. Finally, two of us (S. I. and T. Y.) would like to thank the Iwanami Fuju-kai for financial support.

${ }^{1}$ M. Creutz, Phys. Rev. Lett. 46, 1441 (1981).

${ }^{2}$ H. Bohr and K. J. M. Moriarty, Phys. Lett. 104B, 217 (1981).

3K. J. M. Moriarty, Phys. Lett. 106B, 130 (1981).

${ }^{4}$ M. Creutz and K. J. M. Moriarty, Phys. Rev. D 25, 1724 (1982).

${ }^{5}$ D. Barkai, M. Creutz, and K. J. M. Moriarty, Nucl. Phys. B225, 156 (1983).

6K. G. Wilson, Phys. Rev. D 10, 2445 (1974).

${ }^{7}$ K. G. Wilson and J. Kogut, Phys. Rep. 12C, 75 (1974).

${ }^{8} \mathrm{~K}$. G. Wilson, in Recent Development in Gauge Theories, edited by G.'t Hooft et al. (Plenum, New York, 1980).

${ }^{9}$ Y. Iwasaki, University of Tsukuba Report No. 118 (to be published).

${ }^{10}$ Y. Iwasaki and T. Yoshié, Phys. Lett. 131B, 159 (1983); S. Itoh, Y. Iwasaki, and T. Yoshié, Phys. Lett. 147B, 141 (1984).

${ }^{11}$ N. Cabibbo and E. Marinari, Phys. Lett. 119B, 387 (1982); M. Okawa, Phys. Rev. Lett. 49, 353 (1982).

12P. Weisz, Nucl. Phys. B212, 1 (1983); P. Weisz and R. Wohlert, Nucl. Phys. B236, 397 (1984).

${ }^{13} \mathrm{~K}$. Symanzik, in Mathematical Problems in Theoretical Physics, edited by R. Schrader et al. (Springer-Verlag, Berlin, 1982); Nucl. Phys. B226, 187, 205 (1983).

${ }^{14}$ K. C. Bowler, A. Hasenfratz, P. Hasenfratz, U. Heller, F. Karsch, R. D. Kenway, I. Montvay, G. S. Pawley, D. J. Wallace, CERN-Report No. TH.3952, 1984 (to be published).

${ }^{15} \mathrm{~S}$. Itoh, Y. Iwasaki, and T. Yoshié, University of Tsukuba Report No. 134 (to be published). 